$1 / 5 /\left.\right|_{\text {LA-7034-MS }} ^{81}$

Informal Report
UC.32 and UC-34c

Issued: November 1977

\title{
Data Analysis Facility at LAMPF
}

\author{
Dennis G. Perry \\ James F. Amann \\ Harold S. Butler \\ Cyrus J. Hoffman \\ Richard E. Mischke \\ E. Brooks Shera \\ Henry A. Thiessen
}

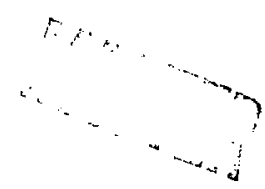




\section{CONTENTS}

$\begin{array}{ll}\text { ABSTRACT } & 1\end{array}$

I. PURPOSE OF THE STUDY AND PARTICIPANTS 1

II. SUMMARY OF RECOMMENDATIONS 2

III. INTRODUCTION 2

IV. HISTORY OF CATA ANALYSIS PROBLEM 2

A. Past Studies 3

B. Incompatibilities with CCF Operation 3

1. Access to the CCF 4

2. Security 4

3. Allocations 4

4. Magretic Tapes 5

V. ANALYSIS OF REQUIREMENTS

A. Past Estimates 5

B. EPICS/HRS 6

C. General Experience 6

D. Magnetic Tape 7

E. Interactive Graphics 7

F. Memory Size 7

G. Projection of Experience 7

VI. SOLUTION TO REQUIREMENTS 7

A. Minicomputer Plus 8

i. Fast Array Processor (FAP) 9

?. FASTBUS Memory 9

3. Cache Memory 9

4. Fast Floating-Point Processor 9

B. Midicomputer 9

C. Operational Costs 9

1. Building Requirements 9

2. Support 11

3. Administration 11

VII. CONCLUSION 


\title{
DATA ANALYSIS FACILITY AT LAMPF
}

\author{
by
}

Dennis G. Perry, James F. Amann, Harold S. Butler,

Cyrus J. Hoffman, Richard E. Mischke, E. Brooks Shera, and Henry A. Thiessen

\section{ABSTRACT}

This report documents the discussions and conclusions of a study held in July 1977 to develop the requirements for a data analysis facility to support the experimental program in medium-energy physics at the Clinton P. Anderson Meson Physics Facility (LAMPF).

\section{PURPOSE OF THE STUDY AND PARTICIPANTS}

This report documents the recommendations of a study group appointed to assess the current status of the data analysis facilities available for the direct support of the experimental program at the Clinton P. Anderson Meson Physics Facility (LAMPF), and as a consequence of $i$ ts assessment, to recommend policies and a course of action for the future which will help make the most effective use of the resources of LAMPF and its user community.

The participants in this study fall into two groups. A larger group provided background information for a laller data analys is comittee which was responsible for the final recommendations. The people invited to this study were the following: *J. Amann, Carnegie-Mellon University; M. Barnes, LAMPF; P. Berardo, LAMPF; *P. Bevington, Case-Western Reserve University; J. Bradbury, LAMPF; *!.. Butler, LAMPF; F. Cverna, Case-Western Reserve University; F. Dorr, LASL; K. Hanson, LAMPF; ${ }^{*}$ C. Hoffman, LAMPF; C. Hwang, LAMPF; R. Jameson, LAMPF; S. Johnson, LAMPF; M. Kellogg, LAMPF; T. Kozlowski, Brookhaven National Laboratory; L. Mann, LASL; *R. Mischke, LAMPF; C. Nelson, LASL; *0. Perry, LAMPF; *E. Shera, LASL; J. Sicilian, LASL; D. Simmonds, LAMPF; N. Spencer, LAMPF; *H. Thiessen, LAMPF; and S. Zink, LAMPF. Those names marked with an asterisk constitute the data analysis committee.

Questions or comments regarding this report should generally be addressed to D. G. Perry or H. S. Butler. 


\section{SUMMARY OF RECOMMENDATIONS}

The recommendations of the study group are listed below and discussed in depth in the sections which follow. These recommendations are expected to satisfy the needs of experimental data analysis as presently understood and projected into the next three to five years. If circumstances change rapidly, then these recommendations shoult be reviewed again and amended appropriately.

A. Sufficient computing power shuuld be obtained to meet the estimated data analysis load of LAMPF. This may be accomplished with the following computer systems:

1. Begin with the PDP-11/70 purchased in FY 77.

2. In FY 78, purchase sufficient equipment to upgrade two computers--one to an enhanced PDP-11/70 for use at HRS/EPICS and another to a normal PDP-11/70. A third PDP-11/70, or a new 32-bit computer, should be ordered.

3. In FY 79, the 32-bit computer should be enhanced with additional peripherals and a second, matching system should be ordered.

4. A midicomputer or its equivalent in computing power and physical characteristics should be ordered as soon as possible, but arrival time should be no later than the first of FY 80.

B. A building should be built to house the midicomputer and the other data analysis computer systems. This building should be located near the experimental area and should include work space for users, offices for computer support personnel, and storage space for magnetic tapes and supplies.

C. The staff of personnel who support the data acquisition and analysis effort-programmers, consultants, operators, and data analysts--should be increased at a minimum by two additional people in $F Y 78$, three additional yeople in FY 79, and by two additional people in FY 80.

\section{INTRODUCTION}

f 5 LAMPF has developed, two study groups have been corivened to analyze the proble ns associated with the acquisition and reduction of experirental data and to recummend guidelines for developing the needed facilities. These recommendations were made jointily by, and had the endorsement of, LAMIPF users and staff.

Since the last study group met in August 1976, LAMPF has increased its output of experimental data considerably. HRS and EPICS have go ie into operation and are producing large volumes of data. The other beam charnels have become more prolific. The data analysis problem as foreseen only a year ago has changed because of unforeseen circumstances. Therefore, it was necessary to convene another study group to review the current status of data anillysis facilities and to plan for their continued expansion. This study was held at LAMPF on July 18 and 19, 1977, with a concluding session on August 4.

\section{HISTORY OF DATA ANALYSIS PROBLEM}

The analysis of experimental data is a preeminent problem at all of the major accelerator centers engaged in nuclear physics research. LAMPF is no exception. 


\section{A. Past Studies}

The problem of data analys is at LAMPF was addressed formally for the first time in 1970. A study group recommended that LAMPF rely on the LAS: CCF for its bulk computing needs, i.e., the computing capacity needed for data analys is. ${ }^{1}$ The capacity required was projected to be equivalent to one CDC-6600 within one or two years of startup and equivalent to two $C D C-6600$ 's by the time all facilities were fully operational.

Those projections were made at a time when the CCF had an inventory of two CDC-7600's and three CDC-6600's. This computing capacity was expected to grow at an annual rate of $40 \%$. Unfortunately, despite the best efforts of the C-Division management, the rate of growth was barely a third of that projected and allocations became a way of life.

In the face of decreasing allocations of computing time and a growing inventory of data tapes, a second study group was convened at LAMPF in 1976. This group recommended that LAMPF augment the CCF resources with a pair of PDP-11s configured for maximum data anaiysis capability. ${ }^{2}$ This recommendation was based on tests that demonstrated the PDP-11 was a cost-effective vehicle fun reducing data. The idea was to conserve the allocation of CCF time for the final stages of data analysis, which are computation intensive, and for calculations related to accelerator development. In anticipation of increased competition for CCF resources, i.e., the rumored departure of one $\operatorname{CDC}-7600$, and the projected growth in the experinental programs at LAMPF, the group further reconmended the acquisition of a medium-sized computer system in FY 79.

\section{B. Incompatibilities with CCF Operation}

In the year since the last study, the pressures from the data-analys is problem have continued to increase. HRS and EPICS, the two most prolific data producing facilities at LAMPF, have become operational. Unfortunately, no new computers have been added to the CCF to handle this increased load. On the contrary, the LAMPF allocation has been decreased. This fact is all the more significant because the experience gained last year established that the analys is of data from a certain class of experiments requires a larger word-length machine than the 16-bit PDP-11, thus making the CCF computers more attractive.

But the most important development from last year's experience was the recognition that there is a basic iricompatibility associated with doing data analysis at the CCF. This observation is not a criticism of the CC.F. The LASL CCF was created to fulfill a certain mission, and that mission dictated a complement of equipment and a philosophy of operation that are incompatible with the requirements for data analysis at LAMDF.

To be more specific, the equipment and operation of the CCF are optimized for large-scale calculations. In contrast, the core of tise data analys is task at LAMPF is processing magnetic tapes. A large computing center can be optimized for this task as has been shown by the Fermi National Accelerator Laboratory. However, it is not appropriate to expect the LASL CCF to alter the parameters of its operation to satisfy the criteria for data analysis. 
This fundamental incompatibility is compounded by four factors which are discussed in the following sections. No single factor by itself totally justifies the conclusion that the analys is of LAMPF data cannot be done reasonably at the CCF. However, all four factors interact in such a way as to create insurmountable difficulties.

1. Access to the CCF. The LASL CCF is located four miles west of LAMPF. This distance impedes access to the CCF because of this shortage of vehicles and limited parkinj. Yet many trips are required to transport the magnetic tapes to the CCF and return them to the users. In addition to the logistics of handling the magnetic tapes, there is a nonnegligible probability of losing tapes, thereby diluting the quality of an experiment. The move by the CCF to a time-sharing environment, while commendable in general, aggravates the problem of coordinating tapes with jobs. The net effect of a remote CCF is to reduce the user's efficiency over what it would be in a freely accessibie, local facility.

2. Security. The LASL CCF operates on a "secure" environment to protect the classified information in the computers. As a consequence, the only personnel who may have indirect access to all of the LASL computers are those with a DOE "Q" clearance. Incleared persons at LASL are restricted to a single CDC- 6600 and may access it only through remote terminals. These security precautions not only hamper the uncleared user, they impose additional burdens on those who have clearances, so much so that essentia? iy all users have given up trying to use the CCF for data znalysis. And it appears that the security regulations will get worse before they get better; for example, the machine for unclassified work will probably not be integrated into the common file system, a decision which will further curtail the computing activities of uncleared uscrs.

3. Allociations. The third factor which is curtailing the data analys is effort is the imited allocation of computing resources granted to LAMPF by the CCF. The current allocation is 33 minutes/day on one CDC- 7600 and 31 minutes on another. These two machines operate under LTSS, a time-sharing operating system. on the third CDC-7600, LAMPF is allocated $2.06 \%$ of the weekly points--ariother measure of allocation. An additional hour/day of CDC-6600 time is available under the NOS operating system. Thus, the total CCF allocation is equivalent to one quarter of a CDC-6600 at a time when the needed capacity is four to eight times that allocation.

The CCF allocation to MP Division is supposed to support all of the computing activities at LAMPF, not just data analysis. A review of present usage disclosed that the current CCF allocation is almost totally invested in accelerator design and performance calculations, data base management, and theoretical nuclear physics calcuiations; very little is left over for data analysis. And the pressures for non-data-analysis calculations are growing.

This discussion of allocations is not meant to be critical of the CCF. The primary mission of the CCF is the support of programmatic weapons development work. As these programmatic requirements have grown, there has not been a corresponding growth in the CCF computing capacity because funds were not available. As a consequence, the CCF capacity was oversubscribed by $8 \%$ in FY 77 and is predicted to be oversubs:ribed by $20 \%$ in FY 78 according to C Division. Thus, there appears to be no relief in sight for the problem of allocations. 
4. Magnetic Tapes. Data analysis at LAMPF invo?ves the processing of 100 to 500 magnetic tapes per experiment. Depending on the type of analys is and the way in which the data were recorded, a single computer run may process ore or more tapes, or multiple runs may be required for a sirigle tape. In any case, the processing is highly interactive and result driven so that the order in which the tapes are processed is not necessarily predictable. This fact dictates that the tapes should be stored at the CCF if they are to be analyzed on the CCF computers. However, the CCF does not have the tape storage capacity for the 10000 or more tapes that may be in various stages of analysis at any given time. The alternative is to transport the tapes to the CCF; however, that has been shown to be impractical, especially' since the current security regulations require that a signed statement be attached to each magnetic tape assuring that the tape contains no classified data. Because of the lack of storage for outside tapes, locating a given tape for a computer run is often difficult. Moreover, the mounting of tapes requires additional effort on the part of already overworked operators and, consequently, jobs involving tapes are subject to long delays. Thus, the proper and speedy handling of magnetic tapes, which is crucial to an efficient data analysis facility, is incompatible with the present boundary conditions on the operation of the CCF.

\section{ANALYSIS OF REQUIREMENTS}

Before proposing solutions to the data analysis problem at LAMPF, it is necessary to document the requirements that must be met.

\section{A. Past Estimates}

The summer study of 1976 surveyed 30 experiments typical to LAMPF to estimate the magnitude of future requirements for data analysis. They projected a total of 10000 to 15000 magnetic tapes of data per year from LAMPF. Since each tape holds a few $\times 10^{8}$ bits of data, LAMPF must be prepared to reduce a few $\times 10^{12}$ bits of data per year given the present array of beam ports. The experience of other accelerator centers indicates that a CDC- 6600 can analyze about $10^{5}$ bits $/ \mathrm{s}$. Given the number of effective seconds of CDC-6600 time in a year, LAMPF was projected to need an in-house computing capacity equivalent to one CDC-6600.

The study group then addressed the alternatives for acquiring this capacity. By analyzing the same data tapes on a CDC-6600 and a PDP-11/45, it was determined that one CDC-6600 was equal to five to six PDP-11/45's. On the other hand, when actual costs for reducing data on the two types of machines were compared, it was determined that tapes could be analyzed for about half the CDC-6600 cost on a PDP-11/45 because of proportionately lower operating and maintenance costs. Consequently, the group recommended that LAMPF acquire, as soon as possib?e, two PDP-11/70 class computers and a permanent building to house a data analys is facility. (The PDP-11/70 was specified because it was the highest performance model of the PDP-11 line.)

Moreover, the group recommended that $\$ 300 \mathrm{~K}$ be budgeted in FY 79 for a major enhancement of the data analysis facility in recognition of the anticipated growth in computing needs as the intersity of the accelerator approached $1 \mathrm{~mA}$. 
Unfortunately, these recommendations came after the Fy 77 budget allocations had been fixed. The best that could be done was to order one PDP-11/70 for delivary in 1977. No funds were available for a building, so the computer will have to be put in the Terminal Building. A second PDP-i1/70 was budgeted for FY 78.

Recently, it has been recognized that while the PDP- $11 / 70$ is suitable for the type of analysis used for the estimates last summer, there is another typical class of data analys is codes that needs a larger and faster main frame.

\section{B. EPICS/HRS}

EPICS and HRS are two of the largest producers of data at LAMPF. EPICS presently can produce data up to a rate of 100 events/s. The PDP-11/45 at EPICS can analyze about 30 events/s when it is available for data analysis. As utilization of EPICS increases, the data acquisition computer will no longer be ayailable for datz analysis. It is anticipated that ihe data event rate for data acquisition will drop somewhat to about 20 events/s because of the lower counting rates typical of pion experiments.

HRS presently produces data up to a rate of 100 events/s. This rate is expected to increase to about 400 events/s with buffered TDC's and ADC's. The HRS PDP-11/45 can analyze about 40 events/s when available.

EPICS and HRS combined need a data analysis facility capable of analyzing data at the rate of 500 events/s or greater. (It is assumed that a $50 \%$ datataking efficiency due to machine and experiment downtime is cancelled by a need to replay some tapes more than once.) The data analysis facility also requires large amounts of memory (a minimum of 256-K bytes), disk space (a minimum of 50-M bytes), interactive graphics, and the other usual assortment of peripherals required to effectively use a computer.

In addition to the type of analysis described above, i.e., replay, a large amount of time must be spent combi.ing runs, finding areas of peaks, subtracting backgrounds, and normalizing results. Typically, this work takes as many man hours as the replay but requires far less CPU time. This is best done in a time-shared mode and is a prime candidate for moving to a larger time-shared machine.

\section{General Experience}

An analysis of users' experience with data acquisition has shown that acquisition event rates may vary from 1 to 400 events/s. Each event may consist of 1 to 200 words of 12-bit data. The number of tapes generated per experiment may vary from 10 to 500 . The time required to analyze these tapes may vary from 1 to $5 \mathrm{~h} /$ tape on a PDP-11/45. From this, one can determine that the time required to analyze a typical experiment may vary from 200 to $1000 \mathrm{~h}$ with a nominal value being $500 \mathrm{~h} / \mathrm{nxperiment}$. One can summarize the requirements for these experiments as requiring a moderately fast computer capable of handling large amounts of data on magnetic tape.

When the nominal EPICS/HRS requirements are added to the estimates from all other channels that will be analyzed at LAMPF, the total requires the approximate 
equivalent computing power of two $C D C-6600$ 's to be available today with an anticipated growth $b^{\circ}$ another factor of 2 .

\section{Magnetic Tape}

One of the major problems of data analysis is the handling and storing of large volumes of magnetic tapes. It is estimated that each experiment will produce from 100 to 500 magnetic tapes. Any data analys is facility must be able to handle tapes from about 60 experiments per year, or anywhere from 5000 to 30000 magnetic tapes per year, with 15000 being a nominal number.

\section{E. Interactive Graphics}

Another prime requirement for data analysis is the ability to display and plot results. The ability to interactively display small portions of data while adjusting selection criteria is invaluable in saving data processing time. After final selection criteria, production runs may proceed with plots needed of the reduced daca.

\section{F. Memory Size}

Many analysis programs require more than the. $32-K$ word limit imposed by the architecture of the PDP-11 computer. In some cases, this may be circumvented by clever programming and segmentation, but in many cases it proves to be a real i imit. These cases require memory sizes which are more typical of larger computers, e.g., 256-K bytes per program task.

\section{G. Projection of Experience}

Our experience with making projections has indicated that it is difficult to project requirements when data acquisition technology is changing so rapidly that the amount of data that can be collected changes by an order of magnitude in a few years. It is also difficult to project how these needs can be met when their fulfillment depends on plans one cannot influence. A greater degree of control may be had with one's own facility.

Our understanding of the data analysis problem indicates that a solution may be obtained with a modest amount of capital expenditure phased over several years. We also anticipate that a greater volume of data will be acquired as future fast-buffered systems are realized. This increase is projected to be close to a factor of 2 beyonc, what is required today.

\section{SOLUTION TO REQUIREMENTS}

A comparison of several representative computers and a measure of their performance is given in Table I. One measure of performance is derived from the Whetstone Benchmarks. ${ }^{3}$ In the Whetstone system, the program is first translated into an intermediate instruction code, which is then executed by an interpretive program. The results of analysis of the Benciriark output are given in kilo-Whetstone instructions per second (kWI/s). Another measure of performance is the Normalized Sarvice Unit (NSU) used by DOE. The NSU is based on the available clock time of a CDC-7600. The whetstone Benchmark is perhaps more 
applicable to our situation and mix of jobs since it more closely represents our situation. To satisfy the data analys is needs of LAMPF requires a facility with at least $3600 \mathrm{kWI} / \mathrm{s}$ by FY 78 and a computer with certain physical characteristics to be available in FY 80 .

TABL.E I

COMPUTER COMPARISON

\begin{tabular}{|c|c|c|c|c|c|c|}
\hline Computer & NSU & $\mathrm{kWI} / \mathrm{s}$ & $\begin{array}{c}\text { Norma lized } \\
\mathrm{k} \text { WI/s } \\
\end{array}$ & $\begin{array}{c}\text { Estimated } \\
\text { Capital Cost } \\
(\mathrm{K} \$) \\
\end{array}$ & $\begin{array}{c}\text { Cost } \\
N S U \\
(K \$) \\
\end{array}$ & $\begin{array}{c}\text { Cost } \\
\text { NKHI/s } \\
\text { (K\$) }\end{array}$ \\
\hline $\begin{array}{l}\text { CDC-7600 } \\
\text { CDC-6600 } \\
\text { DEC } 10 \\
\text { DEC 20 } \\
\text { PDP- } 11 / 70+ \\
\text { PDP- } 11 / 70 \\
\text { PDP- } 11 / 60 \\
\text { PDP- } 11 / 45 \\
\text { PDP- } 11 / 34\end{array}$ & $\begin{array}{c}1.0 \\
0.22 \\
0.16 \\
- \\
-\overline{16} \\
\overline{-} \\
0.07 \\
-\end{array}$ & $\begin{array}{r}8000 \\
1800 \\
1000 \\
1000 \\
1000 \\
700 \\
600 \\
245 \\
45\end{array}$ & $\begin{array}{l}1.0 \\
0.23 \\
0.13 \\
0.13 \\
0.13 \\
0.09 \\
0.08 \\
0.03 \\
0.02\end{array}$ & $\begin{array}{r}7000 \\
2000 \\
1000 \\
500 \\
200 \\
150 \\
100 \\
100 \\
75\end{array}$ & $\begin{array}{c}7000 \\
9091 \\
6250 \\
- \\
- \\
938 \\
- \\
1429 \\
-\end{array}$ & $\begin{array}{l}7000 \\
8696 \\
7692 \\
3846 \\
1538 \\
1667 \\
1250 \\
3333 \\
3750\end{array}$ \\
\hline
\end{tabular}

Since some fraction of data analysis (such as theoretical curve fitting) involves the running of very large codes, it would be useful to have a computer that would run these codes. Certain other types of data analys is also require large codes (such as ray tracing). All data analysis codes appear to be computebound, and the speed of these machines is an advantage for compute-bound jobs. A possible solution is to choose a CDC -7600 or some multiple number of CDC-6600 computers. The acquisition of such a large computer, however, has several disadvantages. One is the very high purchase and maintenance costs in comparison to their performance. Another disadvantage is that large machines require carefully controlled environments, which means substantial costs for a building and its maintenance. It is also estimated that its operating costs would be very high; at least six to eight full-time personnel would be required to operate and manage such a facility. In addition, much of the software used for data acquisition would have to be rewritten for data analysis. Also, the lead time for procurement of a major computer system is prohibitive.

With this in mind, it is apparent from Table I that there exist more costeffective solutions to the data analys is problem at LAMPF. One that this section will discuss is based on a medium-sized computer coupled with several minicomputers with special hardware to enhance their performance.

\section{A. Minicomputer Plus}

It is estimated that most of the EPICS and HRS data analys is can be done on a minicomputer complex with the equivalent computing power of 1600 tc $2000 \mathrm{kWI} / \mathrm{s}$. It is proposed that this need be met with an enhanced PDP-11/70 computer that would provide about $1000 \mathrm{kWI} / \mathrm{s}$. This machine (to be delivered in FY 78) in conjunction with a PDP-11/70 ( $2700 \mathrm{kWI} / \mathrm{s}$ ) delivered in FY 77 and another PDP-11/70 or 32-bit computer to be delivered in FY 78 would give a computing power of about 
$2400 \mathrm{kWI} / \mathrm{s}$, or more than half the total required for LAMPF. As buffered fast acquisition becomes a reality, the needs of EPICS/HRS could double.

Ways of enhancing a PDP-11/70 or a PDP-11/45 to increase its performance are discussed below. Any or all of these ideas may need to be implemented. It should be noted that the speed factors cannot be multiplied together to gain even more speed.

1. Fast Array Processor (FAP). Fast array processors are commercially available, which would be used to speed up certain processes within the dataanalysis program. It is estimated that one could expect a factor of 2 increase in speed on a PDP-11/70 for certain applications. The estimated cost of the increase in speed is $\$ 30$ to $\$ 50 \mathrm{~K}$.

2. FASTBUS Memory. The PDP-11/45 and 11/55 computers have an additional bus besides the UNIBUS. This bus, called the FASTBUS, can be used to add very fast memory to the system. This fast memory can be expected to increase the speed of a PDP-11/45 or a PDP- $11 / 55$ by a factor of 2 . The cost for this increase is about $\$ 10 K / 32-K$ words of memory, or $\$ 40 K$ for a $124-K$ word system.

3. Cache Memory. If one does not want to add fast memory to the FASTBUS, cache memory may be added to the $11 / 45$ or $11 / 55$ to speed up access to memory on the UNIBUS. Depending on the design of the cache and the program running on the machine, a speedup of about a factor of 1.5 may be expected. The cost for cache memory is a total of $\$ 5 \mathrm{~K}$ per machine.

4. Fast Floating-Point Processor. Another alternative to speeding up FORTRAN programs is a fast floating-point processor. These may be added to 11/70, $11 / 60$, 11/55, and $11 / 45$ computers. The expected speedup is about a factor of 2 , depending on how much floating-point calculation is done in the FORTRAN program. The incremental cost is about $\$ 5 \mathrm{~K}$ per machine.

B. Midicomputer

The requirement to run large codes required for certain classes of data analysis makes the choice of a medium-sized computer attractive. in fact, this requirement overcomes the disadvantages of the higher operating cost-performance ratio. Since the minicomputers only provide about $2400 \mathrm{kWI} / \mathrm{s}$, an additional 1000 to $1200 \mathrm{kWI} / \mathrm{s}$ are required to meet the anticipated data analys is needs of LAMPF.

These additional $1000 \mathrm{kWI} / \mathrm{s}$ may be obtained by the use of a class of machine characterized by a DEC System 20 with a price of about $\$ 825 \mathrm{~K}$. A configuration that would meet the needs of data analysis is proposed in Table II.

C. Operational Costs

Unfortunately, the costs of doing data analysis do not erid with the purchase of the hardware required to do the job. Additional costs incurred are building requirements, facilities, administration, and operational support.

1. Building Requirements. The PDP-11/70 to be delivered in FY 77 can be housed in the Terminal Building although with a large degree of discomfort. It is anticipated that the air conditioning of this building will be inadequate and additional cooling will be required. Also, some work must be done to reduce 
TABLE II

COMPUTER CONFIGURATION

Cost

DEC 20/50-C

256-K words memory

LA 36 console

RP06 controller plus two drives

TU45 controller plue one drive

16 communication lines

503000

Mãintenarice

(\$/month)

2773
One additional RP06 drive

Twe additional TU45 drives

Line printer $(1200 \mathrm{c} / \mathrm{min})$

Card reader (300 c/min)

Terminals

Four 4010 \& $\$ 5 \mathrm{~K}$

Twelve VT52 a $\$ 1800$

Network comunications package

PDP-11/34 iront enid

One additional RKO5 drive

Large disk system for $11 / 34$

KS90 for 729 7-track drives for $11 / 34$

Three IBM 729 drives

Plotter, $x y-36$-in. drum for DEC 20 plus software

Networking hardware for $11 / 34$

36650

28000

46200

6170

20000

21600

35000

34640

5600

25000

7000

$-$

31500

1400

Sub total

801760

Software

$$
\text { RSX-11D license }
$$

F4P license

DEC 20 FORTRAN lincense

DECNET license DEC 20

DECNET license $17 / 34$
4200

3000

8250

5000

2750

Total cost 824960

5228

Lease purchase option on 60-month payback

$2.5 \%$ of purchase price/month - $\$ 20624$ 
the noise level of this building as it is currently toc high for comfortable working conditions. These conditions must be rectified in FY 78 . The proposed PDP-11/70 for FY 78 can be housed in the HRS counting room with little adfitional cost. If an additional PDP-11/70 or 32-bit computer is acquired in FY 78 and FY 79 , additional space will be required.

The acquisition of a midicomputer in FY 80 or sooner will also require additional space. It is recommended that this space be located near the experimental area and provide space for all the data analysis computers. Space should also be provided for consultants and experimenters along with permanent magnetic tape storage.

2. Support. The support of the data analys is facility is a large operational cost. This support involves the maintenance of the computers, software support, consulting, and operation of the computer.

The number of additional support personnel is estimated to be two to four, depending on the support level required. An operator/consultant is required for each two PDP-11/70 systems. In addition, the midicomputer would require about three people. This is based on the experience of CTR-6, which runs a large PDP-10 installation. They have one operator, one programmer consultant, and one systems programer. CTR-6 feels that this is the minimum to run their system efficiently and would like to add an additional support operator/technician.

3. Administration. With a distributed data analysis facility, it is important that close administrative control be exercised. This involves usage control, perhaps through a recharge system, so that most efficient use of the facility may be made by arl groups. Also, the direction and supervision of operators, consultants, and programmers required to support the facility is best administered from a single point. Though the location of various computers might be distributed and people assigned to meet the needs of special interests, effective control of the facility san only be accomplished by a single administrator.

Another aspect of administrative controi is the assigning of priorities to certain types of computing. It is presently felt that the performance of the midicomputer should be optimized for time-sharing small jobs during the prime shift and reoptimized for production runs in the two after-hours shifts.

\section{CONCLUSION}

Although it is hoped that the study has arrived at conclusions that will satisfy the requirements for LAMPF's data analysis problem, one should be aware that changing circums tances may alter those requirements. This report addresses only the problem of data analysis, but resources are also needed for continuing support of the data acquisition systems with upgrading of computers and development of new data acquisition hardware.

This summer study of 1977 recognizes these requirements and recommends that the data analysis facility development not be accomplished at the expense of, but in cooperation with, these other vital developments. 


\section{REFERENCES}

1. H. L. Anderson, P. R. Bevington, J. A. Buchanan, H. S. Butler, R. i. Chrien, F. A. Kirsten, J. N. Snyder, and R. F. Thomas, "LAMPF Data-Acquisition System," Los Alamos Scientific Laboratory report LA-4504-MS (August 1970).

2. R. F. Thomas. J. F. Amann, H. S. Butler, J. M. Gallup, R. H. Jeppesen, S. S. Johnson, W. K. McFarlane, M. W. McNaughton, R. E. Mischke, D. G. Perry, M. M. Preedom, R. J. Ridge, E. B. Shera, and H. A. Thiessen, "Computing in Support of Experiments at LAMPF," Los Alamos Scientific Laboratory report LA-6543-MS (October 1976).

3. Harry J. Curnow, in Benchmarking, Nicholas Benwel1, Ed. (Hemisphere Publishing Corp., Washington, 1975), pp. 99-114. 